\title{
New degenerate Bernoulli, Euler, and Genocchi polynomials
}

\author{
OrLI HeRsCOVICI \\ Department of Mathematics \\ Technion - Israel Institute of Technology \\ 3200003 Haifa, Israel \\ email: orli.herscovici@gmail.com \\ and \\ TOUFIK MANSOUR \\ Department of Mathematics \\ University of Haifa \\ 3498838 Haifa, Israel \\ email: tmansour@univ.haifa.ac.il
}

(Received: January 26, 2019, and in revised form May 27, 2019.)

\begin{abstract}
We introduce new generalizations of the Bernoulli, Euler, and Genocchi polynomials and numbers based on the Carlitz-Tsallis degenerate exponential function. Also, we present generalizations of some familiar identities and connection between these types of Bernoulli, Euler, and Genocchi polynomials. Moreover, we establish new analogues of the Euler identity for degenerate Bernoulli polynomials and numbers.
\end{abstract}

Mathematics Subject Classification(2010). 05A10, 05A30, 11B65, $11 \mathrm{~B} 68$.

Keywords: degenerate Bernoulli polynomials and numbers, degenerate Euler polynomials, degenerate Genocchi polynomials, Euler identity for Bernoulli numbers, Carlitz-Tsallis degenerate exponential function, umbral calculus

\section{Introduction}

The Bernoulli, Euler, and Genocchi numbers and polynomials are closely connected to each other [8]. Their study has attracted the attention of many researchers (see $[3,9,12,15]$ and references therein). The Bernoulli numbers appear, for example, in analysis and number theory. Among different identities related to the Bernoulli numbers is the Euler identity. These numbers are connected to the Riemann zeta function, to trigonometric functions, Stirling and Eulerian numbers. These are only a small part of many other topics. Bernoulli numbers cross the boundaries of a pure theory and appear also in perturbative quantum field theory.

Besides the classical version of the exponential function there are different $q$-analogues and parameterized versions $[1,2,7,9,15,17,21]$. The degenerate versions of the Bernoulli and Euler numbers were defined and studied by Carlitz. They are based on the degenerate exponential function $e_{\lambda, \mu}(t)=(1+\lambda t)^{\mu}$, and the degenerate Bernoulli numbers of Carlitz are defined by the generating

1

DOI: $10.1515 /$ puma-2015-0037

(C) 2020 Herscovici, Mansour. This is an open access article licensed under the Creative Commons Attribution-NonCommercial-NoDerivs License (http://creativecommons.org/licenses/by-nc-nd/3.0/). 
function

$$
\frac{t}{(1+\lambda t)^{\mu}-1}=\sum_{n=0}^{\infty} \beta_{n}(\lambda) \frac{t^{n}}{n !}
$$

with condition $\lambda \mu=1$. Degenerate versions of the Bernoulli, Euler, and Genocchi polynomials were studied by different researchers (see $[11,20]$ and references therein). The umbral calculus associated with the classical exponential function was one of the methods used for the study of these polynomials. However, those degenerate polynomials were defined in terms of exponential functions with classical additive property and studied by techniques of the classical umbral calculus. For example, the degenerate Bernoulli polynomials studied in [20] are defined as

$$
\frac{t}{(1+\lambda t)^{\frac{1}{\lambda}}-1}(1+\lambda t)^{\frac{x}{\lambda}}=\sum_{n=0}^{\infty} \beta_{n}(\lambda, x) \frac{t^{n}}{n !},
$$

where the degenerate Bernoulli numbers are evaluated, as usual, as $\beta_{n}(\lambda)=\beta_{n}(\lambda, 0)$. In the definition of the degenerate Bernoulli polynomials studied in [10], the term $(1+\lambda t)^{\frac{x}{\lambda}}$ was replaced by the regular exponential function $e^{x t}$. Another modification was done in [16]. There even the term $(1+\lambda t)^{\frac{1}{\lambda}}$ was replaced by $(1+\lambda)^{\frac{t}{\lambda}}$.

In 1988, Tsallis [18] proposed a generalization of the concept of entropy. This generalization was based on experimental data and led in 1994 to the definition of a new generalized $q$-deformed exponential function [19]:

$$
e_{q}(x)=(1+(1-q) x)^{\frac{1}{1-q}}
$$

One can easily see that the degenerate exponential function of Carlitz, after substituting $\lambda=1-q$, and the deformed exponential function of Tsallis coincide. The fact that the origin of (1) lies in statistical mechanics, makes the study of this function and, in particular, polynomials defined via this deformed exponential function, only more reasonable. There is a place for a new definition of degenerate Bernoulli polynomials and numbers, and for a study of their properties.

In this work we define new degenerate Bernoulli, Euler, and Genocchi polynomials and numbers and study some of their properties. We establish connections between degenerate versions of the Bernoulli and Euler polynomials by applying a few techniques of umbral calculus associated with the deformed exponential function (1). Our results generalize many of the well-known identities for the classical case. Moreover, we provide a new analogue of the Euler identity for degenerate Bernoulli polynomials and numbers.

This paper is organized as follows. We start from some definitions and properties related to the $q$ deformed exponential function (1). In Section 3 we consider the degenerate Bernoulli polynomials and numbers. Section 4 deals mostly with the degenerate Euler polynomials and numbers and, partially, with the degenerate Genocchi polynomials and numbers. In Section 5 we consider connections between these polynomials and show a way to find connections with other polynomials. This paper is selfcontained; some definitions and theorems of the umbral calculus used in this paper are described in the Appendix. 


\section{Background and definitions}

Throughout the paper, we assume that $q \neq 1$. Let us consider the umbral calculus associated with the deformed exponential function (1). It is easy to see that this Carlitz-Tsallis exponential function (1) is an eigenfunction of the operator $D_{q ; x} \equiv(1+(1-q) x) \frac{d}{d x}$, where $d / d x$ is the ordinary derivative. This exponential function has the following expansion as formal power series (see [5]):

$$
e_{q}(x)=1+\sum_{n=1}^{\infty} Q_{n-1}(q) \frac{x^{n}}{n !}
$$

where $Q_{n}(q)=1 \cdot q(2 q-1) \ldots(n q-(n-1))$. Let us define the sequence $c_{n ; q}$ by

$$
c_{n ; q}= \begin{cases}\frac{n !}{Q_{n-1}(q)}, & n \geq 1 \\ 1, & n=0\end{cases}
$$

Therefore, by using this notation, we can write

$$
e_{q}(x)=\sum_{n=0}^{\infty} \frac{x^{n}}{c_{n ; q}} .
$$

Define $x \oplus_{q} y=x+y+(1-q) x y$ (see Borges [4]) and

$$
(x+y)_{c}^{n}=\sum_{k=0}^{n} \frac{c_{n ; q}}{c_{k ; q} c_{n-k ; q}} x^{k} y^{n-k} .
$$

Then we can state the following result.

Proposition 2.1 For any $x, y \in \mathbb{C}$, we have

$$
\sum_{n=0}^{\infty} \frac{\left(x t \oplus_{q} y t\right)^{n}}{c_{n ; q}}=\sum_{n=0}^{\infty}(x+y)_{c}^{n} \frac{t^{n}}{c_{n ; q}} .
$$

Proof. It follows from (2) that

$$
\begin{aligned}
e_{q}(x t) e_{q}(y t) & =\sum_{n=0}^{\infty} \frac{x^{n} t^{n}}{c_{n ; q}} \cdot \sum_{k=0}^{\infty} \frac{y^{k} t^{k}}{c_{k ; q}}=\sum_{n=0}^{\infty} \sum_{k=0}^{n} \frac{c_{n ; q}}{c_{k ; q} c_{n-k ; q}} x^{k} y^{n-k} \frac{t^{n}}{c_{n ; q}} \\
& =\sum_{n=0}^{\infty}(x+y)_{c}^{n} \frac{t^{n}}{c_{n ; q}}=e_{q}\left((x+y)_{c} t\right)
\end{aligned}
$$

while

$$
\begin{aligned}
e_{q}(x t) e_{q}(y t) & =(1+(1-q) x t)^{\frac{1}{1-q}}(1+(1-q) y t)^{\frac{1}{1-q}} \\
& =\left(1+(1-q)\left(x t+y t+(1-q) x y t^{2}\right)\right)^{\frac{1}{1-q}} \\
& =e_{q}\left(x t \oplus_{q} y t\right)=\sum_{n=0}^{\infty} \frac{\left(x t \oplus_{q} y t\right)^{n}}{c_{n ; q}} .
\end{aligned}
$$


Therefore, by comparing (3) with (4), we obtain that $e_{q}\left(x t \oplus_{q} y t\right)=e_{q}\left((x+y)_{c} t\right)$, or, in more detailed notation,

$$
\sum_{n=0}^{\infty} \frac{\left(x t \oplus_{q} y t\right)^{n}}{c_{n ; q}}=\sum_{n=0}^{\infty}(x+y)_{c}^{n} \frac{t^{n}}{c_{n ; q}},
$$

which completes the proof.

From here on we will use the notation

$$
\left(\begin{array}{l}
n \\
k
\end{array}\right)_{c ; q}=\frac{c_{n ; q}}{c_{k ; q} c_{n-k ; q}}
$$

Clearly, $(x+y)_{c}^{n}=\sum_{k=0}^{n}\left(\begin{array}{l}n \\ k\end{array}\right)_{c ; q} x^{k} y^{n-k}$.

It follows from (2) and (18) that $\left\langle e_{q}(y t) \mid x^{n}\right\rangle=y^{n}$ and, respectively, $\left\langle e_{q}(y t)\right|(p(x)\rangle=p(y)$. Let us define the linear operator $D_{c_{q} ; t}$ as

$$
D_{c_{q} ; t} t^{n}= \begin{cases}\frac{c_{n ; q}}{c_{n-1 ; q}} t^{n-1}, & \text { for an integer } n \geq 1 \\ 0, & n=0 .\end{cases}
$$

Let us denote by $\mathbb{N}$ the set of positive integers. Therefore for all $k \in \mathbb{N}$ such that $k \leq n$, one can easily see that $D_{c_{q} ; t}^{k} t^{n}=\frac{c_{n ; q}}{c_{n-k ; q}} t^{n-k}$.

LEMma 2.2 For any polynomial $p(x)=\sum_{j=0}^{n} a_{j} x^{j}$, we have

$$
\left\langle t^{k} \mid p(x)\right\rangle=c_{k ; q} a_{k}=D_{c_{q} ; x}^{k} p(0),
$$

and, in particular, $\left\langle t^{0} \mid p(x)\right\rangle=p(0)$.

Proof. From (19), we immediately obtain $\left\langle t^{k} \mid p(x)\right\rangle=c_{k ; q} a_{k}$. On the other hand we have

$$
D_{c_{q} ; x}^{k} p(x)=D_{c_{q} ; x}^{k} \sum_{j=0}^{n} a_{j} x^{j}=\sum_{j=k}^{n} a_{j} \frac{c_{j ; q}}{c_{j-k ; q}} x^{j-k}
$$

from which we get $D_{c_{q} ; x}^{k} p(0)=a_{k} \frac{c_{k ; q}}{c_{0 ; q}}$. Substituting $c_{0 ; q}=1$ completes the proof.

Let us now define an inverse operator for the operator $D_{c_{q} ; t}$ as follows:

$$
I_{c_{q} ; t} t^{n}=\int t^{n} d_{c_{q}} t=\frac{c_{n ; q}}{c_{n+1 ; q}} t^{n+1}
$$

Obviously, $I_{c_{q} ; t}\left(D_{c_{q} ; t} t^{n}\right)=D_{c_{q} ; t}\left(I_{c_{q} ; t} t^{n}\right)=t^{n}$. 


\section{Bernoulli polynomials}

We define the degenerate Bernoulli polynomials $\mathcal{B}_{n ; q}(x)$ and numbers $\mathcal{B}_{n ; q}$ as

$$
\begin{aligned}
\frac{t}{e_{q}(t)-1} e_{q}(x t) & =\sum_{n=0}^{\infty} \mathcal{B}_{n ; q}(x) \frac{t^{n}}{c_{n ; q}}, \\
\frac{t}{e_{q}(t)-1} & =\sum_{n=0}^{\infty} \mathcal{B}_{n ; q} \frac{t^{n}}{c_{n ; q}} .
\end{aligned}
$$

The first few values of degenerate Bernoulli polynomials and numbers are listed in Table 1.

\begin{tabular}{lll}
\hline$n$ & $\mathcal{B}_{n ; q}(x)$ & $\mathcal{B}_{n ; q}$ \\
\hline 0 & 1 & 1 \\
1 & $x-\frac{q}{2}$ & $-\frac{q}{2}$ \\
2 & $x^{2}-x+\frac{1}{3}-\frac{q}{6}$ & $\frac{1}{3}-\frac{q}{6}$ \\
3 & $\frac{4(2 q-1) x^{3}-6 q x^{2}-(2 q-4) x-\left(q^{2}-3 q+2\right)}{4(2 q-1)}$ & $-\frac{q^{2}-3 q+2}{4(2 q-1)}$ \\
4 & $\frac{\left(6 q^{2}-7 q+2\right) x^{4}-2\left(2 q^{2}-q\right) x^{3}-\left(q^{2}-2\right) x^{2}-\left(q^{2}-3 q+2\right) x-\frac{1}{30}\left(19 q^{3}-76 q^{2}+94 q-36\right)}{(2 q-1)(3 q-2)}$ & $-\frac{19 q^{3}-76 q^{2}+94 q-36}{30(2 q-1)(3 q-2)}$ \\
\hline
\end{tabular}

Table 1: Degenerate Bernoulli polynomials and numbers.

Let us denote by $\mathcal{B}_{q}$ the umbra of the Bernoulli numbers sequence, that is,

$$
\frac{t}{e_{q}(t)-1}=e_{q}\left(\mathcal{B}_{q} t\right) \Longrightarrow t=e_{q}\left(\mathcal{B}_{q} t\right) e_{q}(t)-e_{q}\left(\mathcal{B}_{q} t\right)
$$

By applying (3), we have

$$
t=\sum_{n=0}^{\infty} \frac{\left(\mathcal{B}_{q}+1\right)_{c}^{n} t^{n}}{c_{n ; q}}-\sum_{n=0}^{\infty} \frac{\mathcal{B}_{n ; q} t^{n}}{c_{n ; q}} .
$$

Thus, $\left(\mathcal{B}_{q}+1\right)_{c}^{n}-\mathcal{B}_{n ; q}=\delta_{1, n}$, which is a generalization of a well-known identity for the classical Bernoulli numbers. From (7) we obtain

$$
\begin{aligned}
\sum_{n=0}^{\infty} \mathcal{B}_{n ; q}(x) \frac{t^{n}}{c_{n ; q}} & =\frac{t}{e_{q}(t)-1} e_{q}(x t)=\sum_{n=0}^{\infty} \mathcal{B}_{n ; q} \frac{t^{n}}{c_{n ; q}} \cdot \sum_{k=0}^{\infty} \frac{t^{k} x^{k}}{c_{k ; q}} \\
& =\sum_{n=0}^{\infty} \sum_{k=0}^{n}\left(\begin{array}{l}
n \\
k
\end{array}\right)_{c ; q} \mathcal{B}_{k ; q} x^{n-k} \frac{t^{n}}{c_{n ; q}}
\end{aligned}
$$

and, by extracting the coefficients of $\frac{t^{n}}{c_{n ; q}}$, we get an analogue of another well known identity for the Bernoulli polynomials. 
Proposition 3.1 For all $n \in \mathbb{N}$, the degenerate Bernoulli polynomials $\mathcal{B}_{n ; q}(x)$ defined by (7) satisfy

$$
\mathcal{B}_{n ; q}(x)=\sum_{k=0}^{n}\left(\begin{array}{l}
n \\
k
\end{array}\right)_{c ; q} \mathcal{B}_{n-k ; q} x^{k}
$$

Moreover, for all $n \geq 0$ and $x \in \mathbb{C}$, we have $\mathcal{B}_{n ; q}(x)=\left(\mathcal{B}_{q}+x\right)_{c}^{n}$ and $\mathcal{B}_{n ; q}(1)-\mathcal{B}_{n ; q}=\delta_{1, n}$.

Note that the identity of Proposition 3.1 can be obtained immediately by observing that $\mathcal{B}_{n ; q}(x) \sim$ $\left(\frac{e_{q}(t)-1}{t}, t\right)$ and applying Theorem 6.1(ii). From (22), we obtain

$$
\frac{t}{e_{q}(t)-1} x^{k}=\mathcal{B}_{k ; q}(x), \quad \text { or } \quad x^{k}=\frac{e_{q}(t)-1}{t} \mathcal{B}_{k ; q}(x) .
$$

By applying (21), we get $t \mathcal{B}_{n ; q}(x)=\frac{c_{n ; q}}{c_{n-1 ; q}} \mathcal{B}_{n-1 ; q}(x)$.

LEMMA 3.2 For any polynomial $p(x)=\sum_{k=0}^{n} a_{k} x^{k} \in \mathbb{C}[x]$, we have

$$
\left\langle\frac{e_{q}(y t)-1}{t} \mid p(x)\right\rangle=\int_{0}^{y} p(u) d_{c_{q}} u \text {. }
$$

Proof. Let us consider the action of this linear functional on a monomial $x^{j}$. From (2), we obtain

$$
\left\langle\frac{e_{q}(y t)-1}{t} \mid x^{j}\right\rangle=\left\langle e_{q}(y t)-1 \mid \frac{1}{t} x^{j}\right\rangle
$$

The operator $\frac{1}{t}$ is the inverse of the operator $t$ defined as $t x^{j}=\frac{c_{j ; q}}{c_{j-1 ; q}} x^{j-1}$. Therefore, by applying $\frac{1}{t}$ to both sides of this equation, we obtain $x^{j}=\frac{1}{t} \frac{c_{j ; q}}{c_{j-1 ; q}} x^{j-1}$ and, thus, $\frac{1}{t} x^{j-1}=\frac{c_{j-1 ; q}}{c_{j ; q}} x^{j}$. So by (10), we have

$$
\left\langle\frac{e_{q}(y t)-1}{t} \mid x^{j}\right\rangle=\left\langle e_{q}(y t)-1 \mid \frac{c_{j ; q}}{c_{j+1 ; q}} x^{j+1}\right\rangle=\frac{c_{j ; q}}{c_{j+1 ; q}} y^{j+1}=\int_{0}^{y} x^{j} d_{c_{q}} x
$$

which, by linearity, completes the proof.

By applying Lemma 3.2 to the polynomials $\mathcal{B}_{n ; q}(x)$ and using $(9)$, we obtain

$$
\begin{aligned}
\int_{0}^{1} \mathcal{B}_{n ; q}(u) d_{c_{q}} u & =\left\langle\frac{e_{q}(t)-1}{t} \mid \mathcal{B}_{n ; q}(x)\right\rangle=\left\langle 1 \mid \frac{e_{q}(t)-1}{t} \mathcal{B}_{n ; q}(x)\right\rangle \\
& =\left\langle 1 \mid x^{n}\right\rangle=\left\langle t^{0} \mid x^{n}\right\rangle=c_{n ; q} \delta_{n, 0} .
\end{aligned}
$$

Moreover, by Proposition 3.1, we have

$$
\int_{0}^{1} \mathcal{B}_{n ; q}(u) d_{c_{q}} u=\int_{0}^{1} \sum_{k=0}^{n}\left(\begin{array}{l}
n \\
k
\end{array}\right)_{c ; q} \mathcal{B}_{n-k ; q} u^{k} d_{c_{q}} u=\sum_{k=0}^{n}\left(\begin{array}{l}
n \\
k
\end{array}\right)_{c ; q} \mathcal{B}_{n-k ; q} \int_{0}^{1} u^{k} d_{c_{q}} u
$$


and, by (6), we obtain

$$
\begin{aligned}
\int_{0}^{1} \mathcal{B}_{n ; q}(u) d_{c_{q}} u & =\left.\sum_{k=0}^{n}\left(\begin{array}{c}
n \\
k
\end{array}\right)_{c ; q} \mathcal{B}_{n-k ; q} \frac{c_{k ; q}}{c_{k+1 ; q}} u^{k+1}\right|_{0} ^{1}=\sum_{k=0}^{n} \frac{c_{n ; q}}{c_{k+1 ; q} c_{n-k ; q}} \mathcal{B}_{n-k ; q} \\
& =\frac{c_{n ; q}}{c_{n+1 ; q}} \sum_{k=0}^{n}\left(\begin{array}{c}
n+1 \\
k
\end{array}\right)_{c ; q} \mathcal{B}_{k ; q} .
\end{aligned}
$$

Hence, by comparing (11) with (12) and bringing into consideration that $c_{1 ; q}=1$, we can state the following result.

Proposition 3.3 For all integer $n \geq 0$, we have $\sum_{k=0}^{n}\left(\begin{array}{c}n+1 \\ k\end{array}\right)_{c ; q} \mathcal{B}_{k ; q}=\delta_{n, 0}$.

REMARK 3.4 This Proposition gives another formulation and proof of Proposition 3.1 at $x=1$.

Let us consider (9). It can be rewritten as $t x^{k}=\left(e_{q}(t)-1\right) \mathcal{B}_{k ; q}(x)$. Therefore, by (19), we obtain

$$
\frac{c_{k ; q}}{c_{k-1 ; q}} x^{k-1}=e_{q}(t) \mathcal{B}_{k ; q}(x)-\mathcal{B}_{k ; q}(x)=\sum_{j=0}^{k}\left(\begin{array}{c}
k \\
j
\end{array}\right)_{c ; q} \mathcal{B}_{k ; q}(x)-\mathcal{B}_{k ; q}(x)=\sum_{j=0}^{k-1}\left(\begin{array}{c}
k \\
j
\end{array}\right)_{c ; q} \mathcal{B}_{k ; q}(x) .
$$

Let $\left[x^{n}\right] p(x)$ denote the coefficient of $x^{n}$ in $p(x)$. Thus we can state the following result.

Proposition 3.5 For all integer $n \geq 0$, we have

$$
x^{n}=\frac{c_{n ; q}}{c_{n+1 ; q}} \sum_{k=0}^{n}\left(\begin{array}{c}
n+1 \\
k
\end{array}\right)_{c ; q} \mathcal{B}_{k ; q}(x) .
$$

Moreover, for all integer $n \geq 0,\left[x^{n}\right] \mathcal{B}_{n ; q}(x)=1$.

Now, we are ready to present an analogue of the Euler identity for Bernoulli polynomials and numbers.

THEOREM 3.6 For all integer $n \geq 2$, the degenerate Bernoulli polynomials defined by (7) satisfy

$$
\begin{aligned}
\sum_{k=0}^{n}\left(\begin{array}{l}
n \\
k
\end{array}\right)_{c ; q} \mathcal{B}_{k ; q}(x) \mathcal{B}_{n-k ; q}(y) & =-(n-1) \mathcal{B}_{n ; q}\left((x+y)_{c}\right)-n \mathcal{B}_{n-1 ; q}\left((x+y)_{c}\right) \frac{(n-1)-q(n-2)}{(n-1) q-(n-2)} \\
& +\widehat{\mathcal{B}_{n ; q}}\left((x+y)_{c}\right)+(1-q) \frac{c_{n ; q}}{c_{n-1 ; q}} \widehat{\mathcal{B}_{n-1 ; q}}\left((x+y)_{c}\right),
\end{aligned}
$$

where $\widehat{\mathcal{B}_{n ; q}}(u)=\sum_{k=0}^{n}\left(\begin{array}{l}n \\ k\end{array}\right)_{c ; q} k \mathcal{B}_{n-k ; q} u^{k}$.

Proof. Let $b(t)=\frac{t}{e_{q}(t)-1}$. Therefore

$$
b(t)^{2}=(1-q t) b(t)-(1+(1-q) t) t b^{\prime}(t)
$$


where $b^{\prime}(t)=\frac{d}{d t} b(t)$. By multiplying both sides by $e_{q}(x t) e_{q}(y t)$ and using $b^{\prime}(t) e_{q}(u t)=\left(b(t) e_{q}(u t)\right)^{\prime}-$ $b(t) e_{q}^{\prime}(u t)$ in accordance with the product rule, we obtain

$$
\begin{aligned}
b(t)^{2} e_{q}(x t) e_{q}(y t) & =(1-q t) b(t) e_{q}\left((x+y)_{c} t\right) \\
& -\left(t+(1-q) t^{2}\right)\left[\left(b(t) e_{q}\left((x+y)_{c} t\right)\right)^{\prime}-b(t) e_{q}^{\prime}\left((x+y)_{c} t\right)\right] .
\end{aligned}
$$

It follows that

$$
\begin{aligned}
\sum_{n=0}^{\infty} \sum_{k=0}^{n}\left(\begin{array}{l}
n \\
k
\end{array}\right)_{c ; q} \mathcal{B}_{k ; q}(x) \mathcal{B}_{n-k ; q}(y) \frac{t^{n}}{c_{n ; q}}=(1-q t) \sum_{n=0}^{\infty} \mathcal{B}_{n ; q}\left((x+y)_{c}\right) \frac{t^{n}}{c_{n ; q}} \\
-\left(t+(1-q) t^{2}\right)\left(\sum_{n=0}^{\infty} \mathcal{B}_{n ; q}\left((x+y)_{c}\right) \frac{t^{n}}{c_{n ; q}}\right)^{\prime} \\
+\left(t+(1-q) t^{2}\right) \sum_{n=0}^{\infty} \mathcal{B}_{n ; q} \frac{t^{n}}{c_{n ; q}} \cdot\left(\sum_{k=0}^{\infty}(x+y)_{c}^{k} \frac{t^{k}}{c_{k ; q}}\right)^{\prime} .
\end{aligned}
$$

After differentiating and applying the Cauchy product, one can extract the coefficients of $\frac{t^{n}}{c_{n ; q}}$ for $n \geq 2$ as

$$
\begin{aligned}
\sum_{k=0}^{n}\left(\begin{array}{l}
n \\
k
\end{array}\right)_{c ; q} \mathcal{B}_{k ; q}(x) \mathcal{B}_{n-k ; q}(y) & =\mathcal{B}_{n ; q}\left((x+y)_{c}\right)-\frac{c_{n ; q}}{c_{n-1 ; q}} q \mathcal{B}_{n-1 ; q}\left((x+y)_{c}\right) \\
& -n \mathcal{B}_{n ; q}\left((x+y)_{c}\right)-\frac{c_{n ; q}}{c_{n-1 ; q}}(1-q)(n-1) \mathcal{B}_{n-1 ; q}\left((x+y)_{c}\right) \\
& +\sum_{k=0}^{n-1}\left(\begin{array}{l}
n \\
k
\end{array}\right)_{c ; q}(n-k) \mathcal{B}_{k ; q} \cdot(x+y)_{c}^{n-k} \\
& +\sum_{k=0}^{n-2}\left(\begin{array}{c}
n-1 \\
k
\end{array}\right)_{c ; q}(n-k-1)(1-q) \mathcal{B}_{k ; q} \cdot(x+y)_{c}^{n-k-1} \frac{c_{n ; q}}{c_{n-1 ; q}}
\end{aligned}
$$

Rearranging the summation indices, denoting $\widehat{\mathcal{B}_{n ; q}}(u)=\sum_{k=0}^{n}\left(\begin{array}{l}n \\ k\end{array}\right)_{c ; q} k \mathcal{B}_{n-k ; q} u^{k}$, and gathering similar terms completes the proof.

An analogue of the Euler identity for the degenerate Bernoulli numbers follows immediately from Theorem 3.6 with $x=y=0$ and noting that $\widehat{\mathcal{B}_{n ; q}}(0)=0$ for all integers $n \geq 0$.

Corollary 3.7 For all integer $n \geq 2$, the degenerate Bernoulli numbers defined by (8) satisfy

$$
\sum_{k=1}^{n}\left(\begin{array}{l}
n \\
k
\end{array}\right)_{c ; q} \mathcal{B}_{k ; q} \mathcal{B}_{n-k ; q}=-(n-1) \mathcal{B}_{n ; q}-n \mathcal{B}_{n-1 ; q} \frac{(n-1)-q(n-2)}{(n-1) q-(n-2)}
$$




\section{Euler and Genocchi polynomials}

We now define degenerate Euler polynomials $\mathcal{E}_{n ; q}(x)$ and values $\mathcal{E}_{n ; q}=\mathcal{E}_{n ; q}(0)$ as

$$
\begin{aligned}
\frac{2}{e_{q}(t)+1} e_{q}(x t) & =\sum_{n=0}^{\infty} \mathcal{E}_{n ; q}(x) \frac{t^{n}}{c_{n ; q}}, \\
\frac{2}{e_{q}(t)+1} & =\sum_{n=0}^{\infty} \mathcal{E}_{n ; q} \frac{t^{n}}{c_{n ; q}} .
\end{aligned}
$$

The first five degenerate Euler polynomials and their special values are listed in Table 2.

\begin{tabular}{lll}
\hline$n$ & $\mathcal{E}_{n ; q}(x)$ & $\mathcal{E}_{n ; q}$ \\
\hline 0 & 1 & 1 \\
1 & $x-\frac{1}{2}$ & $-\frac{1}{2}$ \\
2 & $\frac{2 x^{2} q-2 x-q+1}{2 q}$ & $\frac{1-q}{2 q}$ \\
3 & $\frac{\left(8 q^{2}-4 q\right) x^{3}-6 q x^{2}+(6-6 q) x-4 q^{2}+8 q-3}{4 q(2 q-1)}$ & $\frac{-4 q^{2}+8 q-3}{4 q(2 q-1)}$ \\
4 & $\frac{\left(12 q^{3}-14 q^{2}+4 q\right) x^{4}-\left(8 q^{2}-4 q\right) x^{3}-\left(6 q^{2}-6 q\right) x^{2}-\left(8 q^{2}-16 q+6\right) x-6 q^{3}+18 q^{2}-15 q+3}{2 q(2 q-1)(3 q-2)}$ & $\frac{-6 q^{3}+18 q^{2}-15 q+3}{2 q(2 q-1)(3 q-2)}$ \\
\hline
\end{tabular}

Table 2: Degenerate Euler polynomials and values.

It is easy to see that $\mathcal{E}_{n ; q}(x) \sim\left(\frac{e_{q}(t)+1}{2}, t\right)$. Therefore, by $(21)$, we obtain

$$
t \mathcal{E}_{n ; q}(x)=\frac{c_{n ; q}}{c_{n-1 ; q}} \mathcal{E}_{n-1 ; q}(x) \text { and } D_{c_{q} ; x}^{k} \mathcal{E}_{n ; q}(x)=\frac{c_{n ; q}}{c_{n-k ; q}} \mathcal{E}_{n-k ; q}(x) .
$$

From (22), we have

$$
\frac{2}{e_{q}(t)+1} x^{k}=\mathcal{E}_{k ; q}(x), \text { or } x^{k}=\frac{e_{q}(t)+1}{2} \mathcal{E}_{k ; q}(x) .
$$

Let us denote by $\mathcal{E}_{q}$ the umbra of the sequence of Euler values, that is,

$$
\frac{2}{e_{q}(t)+1}=e_{q}\left(\mathcal{E}_{q} t\right) \Longrightarrow 2=e_{q}\left(\mathcal{E}_{q} t\right) e_{q}(t)+e_{q}\left(\mathcal{E}_{q} t\right) .
$$

By applying (3), we get $2=\sum_{n=0}^{\infty} \frac{\left(\mathcal{E}_{q}+1\right)_{c}^{n} t^{n}}{c_{n ; q}}+\sum_{n=0}^{\infty} \frac{\mathcal{E}_{n ; q} t^{n}}{c_{n ; q}}$. Thus, $\left(\mathcal{E}_{q}+1\right)_{c}^{n}+\mathcal{E}_{n ; q}=2 \delta_{0, n}$, which is a generalization of a well-known identity for the classical case. From (13) and (14), we get

$$
\begin{aligned}
\sum_{n=0}^{\infty} \mathcal{E}_{n ; q}(x) \frac{t^{n}}{c_{n ; q}} & =\frac{2}{e_{q}(t)+1} \cdot e_{q}(x t)=\sum_{n=0}^{\infty} \mathcal{E}_{n ; q} \frac{t^{n}}{c_{n ; q}} \cdot \sum_{k=0}^{\infty} \frac{x^{k} t^{k}}{c_{k ; q}} \\
& =\sum_{n=0}^{\infty} \sum_{k=0}^{n} \frac{c_{n ; q}}{c_{k ; q} c_{n-k ; q}} \mathcal{E}_{n-k ; q} x^{k} \frac{t^{n}}{c_{n ; q}}
\end{aligned}
$$

which leads to the following result. 
Proposition 4.1 For all $n \in \mathbb{N}$, the degenerate Euler polynomials $\mathcal{E}_{n ; q}(x)$ defined by (13) satisfy

$$
\mathcal{E}_{n ; q}(x)=\sum_{k=0}^{n}\left(\begin{array}{l}
n \\
k
\end{array}\right)_{c ; q} \mathcal{E}_{n-k ; q} x^{k} .
$$

Moreover, for all $n \geq 0$ and $x \in \mathbb{C}$,

$$
\mathcal{E}_{n ; q}(x)=\left(\mathcal{E}_{q}+x\right)_{c}^{n} \text { and } \mathcal{E}_{n ; q}(1)+\mathcal{E}_{n ; q}=2 \delta_{0, n} .
$$

From Theorem 6.1 with $y=1$, we obtain

$$
e_{q}(t) \mathcal{E}_{n ; q}(x)=\sum_{k=0}^{n} \frac{c_{n ; q}}{c_{k ; q} c_{n-k ; q}} \mathcal{E}_{k ; q}(x)
$$

Applying this identity to (15) leads to the next result.

Proposition 4.2 For all integer $n \geq 0$, we have

$$
x^{n}=\frac{1}{2} \sum_{k=0}^{n}\left(\begin{array}{l}
n \\
k
\end{array}\right)_{c ; q} \mathcal{E}_{k ; q}(x)+\frac{1}{2} \mathcal{E}_{n ; q}(x) .
$$

Moreover, $\left[x^{n}\right] \mathcal{E}_{n ; q}(x)=1$ for all $n \geq 0$.

By substituting $x=0$ into the statement Proposition 4.2 and rearranging the terms, we obtain the following result.

COROLlary 4.3 For all integer $n \geq 1$, we have

$$
-2 \mathcal{E}_{n ; q}=\sum_{k=0}^{n-1}\left(\begin{array}{l}
n \\
k
\end{array}\right)_{c ; q} \mathcal{E}_{k ; q} .
$$

Let us define degenerate Genocchi polynomials $\mathcal{G}_{n ; q}(x)$ and numbers $\mathcal{G}_{n ; q}$ as

$$
\begin{aligned}
\frac{2 t}{e_{q}(t)+1} e_{q}(x t) & =\sum_{n=0}^{\infty} \mathcal{G}_{n ; q}(x) \frac{t^{n}}{c_{n ; q}}, \\
\frac{2 t}{e_{q}(t)+1} & =\sum_{n=0}^{\infty} \mathcal{G}_{n ; q} \frac{t^{n}}{c_{n ; q}} .
\end{aligned}
$$

By comparing (13) and (16), we can immediately conclude that

$$
\mathcal{G}_{n ; q}(x)=\frac{c_{n ; q}}{c_{n-1 ; q}} \mathcal{E}_{n-1 ; q}(x) .
$$

Therefore relations similar to those obtained for the degenerate Euler polynomials can be easily obtained for the degenerate Genocchi polynomials. 


\section{Connections between polynomials}

We already have shown a connection between monomials $p(x)=x^{n}$ and degenerate Bernoulli $\mathcal{B}_{n ; q}(x)$ and Euler $\mathcal{E}_{n ; q}(x)$ polynomials. For a polynomial $p(x) \in \mathbb{C}[x]$ of degree $n$, write $p(x)=\sum_{k=0}^{n} b_{k} \mathcal{B}_{k ; q}(x)$. Therefore, by Theorem 6.1(i), we obtain

$$
\sum_{k=0}^{n} b_{k} \mathcal{B}_{k ; q}(x)=\sum_{k=0}^{n} \frac{\left\langle\frac{e_{q}(t)-1}{t} \cdot t^{k} \mid p(x)\right\rangle}{c_{k ; q}} \mathcal{B}_{k ; q}(x),
$$

where

$$
\begin{aligned}
b_{k} & =\frac{1}{c_{k ; q}}\left\langle\frac{e_{q}(t)-1}{t} \cdot t^{k} \mid p(x)\right\rangle=\frac{1}{c_{k ; q}}\left\langle\frac{e_{q}(t)-1}{t} \cdot \mid t^{k} p(x)\right\rangle \\
& =\frac{1}{c_{k ; q}}\left\langle\frac{e_{q}(t)-1}{t} \cdot \mid D_{c_{q} ; x}^{k} p(x)\right\rangle=\frac{1}{c_{k ; q}} \int_{0}^{1} D_{c_{q} ; x}^{k} p(x) d_{c_{q}} x .
\end{aligned}
$$

Thus, we can state the following result.

Proposition 5.1 For any polynomial $p(x) \in \mathbb{C}[x]$ of degree $n$, we have

$$
p(x)=\sum_{k=0}^{n} b_{k} \mathcal{B}_{k ; q}(x),
$$

where

$$
b_{k}=\frac{1}{c_{k ; q}} \int_{0}^{1} D_{c_{q} ; x}^{k} p(x) d_{c_{q}} x .
$$

TheOREM 5.2 For all integers $n \geq 0$,

$$
\mathcal{E}_{n ; q}(x)=-2 \frac{c_{n ; q}}{c_{n+1 ; q}} \sum_{k=0}^{n}\left(\begin{array}{c}
n+1 \\
k
\end{array}\right)_{c ; q} \mathcal{E}_{n-k+1 ; q} \mathcal{B}_{k ; q}(x) .
$$

Proof. Let us assume that $\mathcal{E}_{n ; q}(x)=\sum_{k=0}^{n} b_{k} \mathcal{B}_{k ; q}(x)$. Therefore, by Proposition 5.1, we have

$$
\begin{aligned}
b_{k} & =\frac{1}{c_{k ; q}} \int_{0}^{1} D_{c_{q} ; x}^{k} \mathcal{E}_{n ; q}(x) d_{c_{q}} x=\frac{1}{c_{k ; q}} \int_{0}^{1} \frac{c_{n ; q}}{c_{n-k ; q}} \mathcal{E}_{n-k ; q}(x) d_{c_{q}} x \\
& =\left.\frac{c_{n ; q}}{c_{k ; q} c_{n-k ; q}} \cdot \frac{c_{n-k ; q}}{c_{n-k+1 ; q}} \mathcal{E}_{n-k+1 ; q}(x)\right|_{0} ^{1}=\frac{c_{n ; q}}{c_{k ; q} c_{n-k+1 ; q}}\left(\mathcal{E}_{n-k+1 ; q}(1)-\mathcal{E}_{n-k+1 ; q}\right) .
\end{aligned}
$$

From Proposition 4.1, we obtain

$$
b_{k}=\frac{c_{n ; q}}{c_{k ; q} c_{n-k+1 ; q}}\left(2 \delta_{0, n-k+1}-2 \mathcal{E}_{n-k+1 ; q}\right) .
$$

Therefore,

$$
\begin{aligned}
\mathcal{E}_{n ; q}(x) & =2 \frac{c_{n ; q}}{c_{n+1 ; q}} \sum_{k=0}^{n}\left(\begin{array}{c}
n+1 \\
k
\end{array}\right)_{c ; q}\left(\delta_{0, n-k+1}-\mathcal{E}_{n-k+1 ; q}\right) \mathcal{B}_{k ; q}(x) \\
& =-2 \frac{c_{n ; q}}{c_{n+1 ; q}} \sum_{k=0}^{n}\left(\begin{array}{c}
n+1 \\
k
\end{array}\right)_{c ; q} \mathcal{E}_{n-k+1 ; q} \mathcal{B}_{k ; q}(x),
\end{aligned}
$$

which completes the proof. 
Proposition 5.3 For any polynomial $p(x) \in \mathbb{C}[x]$ of degree $n$, we have

$$
p(x)=\sum_{k=0}^{n} b_{k} \mathcal{E}_{k ; q}(x),
$$

where

$$
b_{k}=\frac{1}{c_{k ; q}} \frac{\left(D_{c_{q} ; x}^{k} p\right)(1)-\left(D_{c_{q} ; x}^{k} p\right)(0)}{2}
$$

Proof. Theorem 6.1(i) gives

$$
p(x)=\sum_{k=0}^{n} b_{k} \mathcal{E}_{k ; q}(x)=\sum_{k=0}^{n} \frac{\left\langle\frac{e_{q}(t)+1}{2} t^{k} \mid p(x)\right\rangle}{c_{k ; q}} \mathcal{E}_{k ; q}(x) .
$$

Therefore

$$
\begin{aligned}
b_{k} & =\frac{1}{c_{k ; q}}\left\langle\frac{e_{q}(t)+1}{2} t^{k} \mid p(x)\right\rangle=\frac{1}{c_{k ; q}}\left\langle\frac{e_{q}(t)+1}{2} \mid t^{k} p(x)\right\rangle \\
& =\frac{1}{c_{k ; q}}\left\langle\frac{e_{q}(t)+1}{2} \mid D_{c_{q} ; x}^{k} p(x)\right\rangle=\frac{1}{c_{k ; q}} \frac{\left(D_{c_{q} ; x}^{k} p\right)(1)+\left(D_{c_{q} ; x}^{k} p\right)(0)}{2},
\end{aligned}
$$

as required.

THEOREM 5.4 For all integer $n \geq 1$,

$$
\mathcal{B}_{n ; q}(x)=\sum_{k=0}^{n}\left(\begin{array}{l}
n \\
k
\end{array}\right)_{c ; q} \mathcal{B}_{n-k ; q} \mathcal{E}_{k ; q}(x)+\frac{c_{n ; q}}{c_{n-1 ; q}} \mathcal{E}_{n-1 ; q}(x) .
$$

Proof. Let us assume that $\mathcal{B}_{n ; q}(x)=\sum_{k=0}^{n} b_{k} \mathcal{E}_{k ; q}(x)$. Therefore, by Proposition 5.3, we have

$$
b_{k}=\frac{\left(D_{c_{q} ; x}^{k} \mathcal{B}_{n ; q}(x)\right)(1)+\left(D_{c_{q} ; x}^{k} \mathcal{B}_{n ; q}(x)\right)(0)}{2 c_{k ; q}}=\frac{1}{2 c_{k ; q}}\left(\frac{c_{n ; q}}{c_{n-k ; q}} \mathcal{B}_{n-k ; q}(1)+\frac{c_{n ; q}}{c_{n-k ; q}} \mathcal{B}_{n-k ; q}\right) .
$$

So by Proposition 3.1, we obtain

$$
b_{k}=\frac{1}{2}\left(\begin{array}{l}
n \\
k
\end{array}\right)_{c ; q}\left(\mathcal{B}_{n-k ; q}+\delta_{1, n-k}+\mathcal{B}_{n-k ; q}\right)=\frac{1}{2}\left(\begin{array}{l}
n \\
k
\end{array}\right)_{c ; q}\left(2 \mathcal{B}_{n-k ; q}+\delta_{1, n-k}\right) .
$$

Therefore, we get

$$
\begin{aligned}
\mathcal{B}_{n ; q}(x) & =\sum_{k=0}^{n} \frac{1}{2}\left(\begin{array}{l}
n \\
k
\end{array}\right)_{c ; q}\left(2 \mathcal{B}_{n-k ; q}+\delta_{1, n-k}\right) \mathcal{E}_{k ; q}(x) \\
& =\sum_{k=0}^{n}\left(\begin{array}{l}
n \\
k
\end{array}\right)_{c ; q} \mathcal{B}_{n-k ; q} \mathcal{E}_{k ; q}(x)+\frac{c_{n ; q}}{c_{n-1 ; q}} \mathcal{E}_{n-1 ; q}(x),
\end{aligned}
$$

which completes the proof. 


\section{Appendix - Umbral Calculus}

Let $P$ be the algebra of polynomials in a single variable $x$ over the field $\mathbb{C}$ and $P^{*}$ be the vector space of all linear functionals on $P$. The notation $\langle L \mid p(x)\rangle$ denotes the action of a linear functional $L$ on a polynomial $p(x)$, and the vector space operations on $P^{*}$ are defined by $\left\langle\alpha_{1} L_{1}+\alpha_{2} L_{2} \mid p(x)\right\rangle=$ $\alpha_{1}\left\langle L_{1} \mid p(x)\right\rangle+\alpha_{2}\left\langle L_{2} \mid p(x)\right\rangle$ for any constants $\alpha_{1}, \alpha_{2} \in \mathbb{C}$. Let $\mathcal{F}$ denote the algebra of formal power series in a single variable $t$ over the field $\mathbb{C}$ :

$$
\mathcal{F}=\left\{f(t)=\sum_{k \geq 0} a_{k} \frac{t^{k}}{c_{k}} \mid a_{k} \in \mathbb{C}\right\}
$$

where $c_{k}$ is a sequence of nonzero constants. The formal power series $f(t)$ defines a linear functional on $P$ by setting

$$
\left\langle f(t) \mid x^{n}\right\rangle=c_{n} a_{n}, \quad \text { for all } n \geq 0,
$$

and in particular $\left\langle t^{k} \mid x^{n}\right\rangle=c_{n} \delta_{n, k}$ for all $n, k \geq 0$, where $\delta_{n, k}$ is the Kronecker delta function.

Let $f_{L}(t)=\sum_{k \geq 0} \frac{\left\langle L \mid x^{k}\right\rangle}{c_{k}} t^{k}$; then we get $\left\langle f_{L}(t) \mid x^{n}\right\rangle=\left\langle L \mid x^{n}\right\rangle$. Thus the map $L \mapsto F_{L}(t)$ is a vector space isomorphism from $P^{*}$ onto $\mathcal{F}$. Henceforth, $\mathcal{F}$ denotes both the algebra of formal power series in $t$ and the vector space of all linear functionals in $P$ (see [13]), so that $\mathcal{F}$ is an umbral algebra, and the umbral calculus is the study of the umbral algebra. Note that the umbral calculus considered here is not in the standard form because it is associated with the sequence $c_{n}$ instead of classical $n$ !. For all $f(t) \in \mathcal{F}$ and for all polynomials $p(x) \in P$ we have

$$
f(t)=\sum_{k \geq 0} \frac{\left\langle f(t) \mid x^{k}\right\rangle}{c_{k}} t^{k} \quad \text { and } \quad p(x)=\sum_{k \geq 0} \frac{\left\langle t^{k} \mid p(x)\right\rangle}{c_{k}} x^{k} .
$$

For $f_{1}(t), \ldots, f_{m}(t) \in \mathcal{F}$, we have (see $\left.[13,14]\right)$

$$
\left\langle f_{1}(t) \cdots f_{m}(t) \mid x^{n}\right\rangle=\sum_{\substack{i_{1}+\cdots+i_{m}=n \\ i_{j} \geq 0}} \frac{c_{n}}{c_{i_{1}} \cdots c_{i_{m}}}\left\langle f_{1}(t) \mid x^{i_{1}}\right\rangle \cdots\left\langle f_{m}(t) \mid x^{i_{m}}\right\rangle .
$$

For any $f(t) \in \mathcal{F}$ the linear operator $f(t)$ on $\mathcal{F}$ is defined by (see [13]) $f(t) x^{n}=\sum_{k=0}^{n} \frac{c_{n}}{c_{k} c_{n-k}} a_{k} x^{n-k}$, which, in particular, leads to

$$
t^{k} x^{n}= \begin{cases}\frac{c_{n}}{c_{n-k}} x^{n-k}, & \text { for integer } n \geq k \\ 0, & n<k\end{cases}
$$

For $f(t), g(t) \in \mathcal{F}$, we have $\langle f(t) g(t) \mid p(x)\rangle=\langle g(t) \mid f(t) p(x)\rangle=\langle f(t) \mid g(t) p(x)\rangle$. The order of $f(t)$ (denoted by $o(f(t)))$ is the smallest $k$ such that $t^{k}$ does not vanish. If $o(f(t))=0$ then the series $f(t)$ is called invertible and has a multiplicative inverse denoted by $1 / f(t)$. If $o(f(t))=1$ then the series $f(t)$ is called delta series and has a compositional inverse $\bar{f}(t)$ satisfying $f(\bar{f}(t))=\bar{f}(f(t))=t$. For a delta series $f(t) \in \mathcal{F}$ and an invertible series $g(t) \in \mathcal{F}$ we say that a polynomial sequence $s_{n}(x)$ is 
a Sheffer sequence for the pair $(g(t), f(t))$ and denote it by $s_{n}(x) \sim(g(t), f(t))$ if for all $n, k \geq 0$ we have $\left\langle g(t) f(t)^{k} \mid s_{n}(x)\right\rangle=c_{n} \delta_{n, k}$. Thus, $s_{n}(x) \sim(g(t), f(t))$ if and only if

$$
\frac{1}{g(\bar{f}(t))} \varepsilon(y \bar{f}(t))=\sum_{n=0}^{\infty} \frac{s_{n}(y)}{c_{n}} t^{n} \quad \text { for all } y \in \mathbb{C},
$$

where $\varepsilon(x)=\sum_{n \geq 0} \frac{x^{n}}{c_{n}}$ is a generalized exponential function (see [13]). The following statements are equivalent:

$$
\begin{aligned}
& s_{n}(x) \sim(g(t), f(t)), \\
& g(t) s_{n}(x) \sim(1, f(t)), \\
& f(t) s_{n}(x)=\frac{c_{n}}{c_{n-1}} s_{n-1}(x) .
\end{aligned}
$$

Moreover, the following theorem holds; see Theorems 6.2.3, 6.2.5, and 6.2.8 in [18].

TheOREm 6.1 Let $s_{n}(x) \sim(g(t), f(t))$. Then

(i) for any polynomial $p(x), p(x)=\sum_{n=0}^{\infty} \frac{\left\langle g(t) f(t)^{n} \mid p(x)\right\rangle}{c_{n}} s_{n}(x)$,

(ii) $s_{n}(x)=\sum_{k=0}^{n} \frac{\left\langle g(\bar{f}(t))^{-1} \bar{f}(t)^{k} \mid x^{n}\right\rangle}{c_{k}} x^{k}$.

Moreover, a sequence $s_{n}(x) \sim(g(t), f(t))$ for some invertible $g(t)$, if and only if

$$
\varepsilon(y t) s_{n}(x)=\sum_{k=0}^{n} \frac{c_{n}}{c_{k} c_{n-k}} p_{k}(y) s_{n-k}(x)
$$

for all constants $y$, where $p(x) \sim(1, f(t))$.

Finally, if $s_{n}(x) \sim(g(t), t)$ then, from $(20)$ we obtain

$$
\frac{1}{g(t)} x^{n}=s_{n}(x), \quad \text { or } \quad x^{n}=g(t) s_{n}(x) .
$$

Acknowledgement. We thank the anonymous reviewers for the careful reading of our manuscript and their insightful comments and suggestions. The research of the first author was partially supported by the Ministry of Science and Technology and partially by the Israel Science Foundation (grant No. 1692/17).

\section{References}

[1] M. Acikgoz, S. Araci And U. Duran, New extensions of some known special polynomials under the theory of multiple q-calculus, Turkish J. Anal. Num. Theory, 3 (2015) 128-139.

[2] M. Acikgoz And Y. Simsek, On multiple interpolation functions of the Nörlund type q-Euler polynomials, Abstr. Appl. Anal., 2009 (2009) Article ID 382574, 14pp. 
[3] S. Araci, M. ACIKGoz And E. ŞEn, Some new formulae for Genocchi numbers and polynomials involving Bernoulli and Euler polynomials, Int. J. Math. Math. Sci., 2014 (2014) Article ID 760613,7 pp.

[4] E. P. Borges, A possible deformed algebra and calculus inspired in nonextensive thermostatistics, Phys. A., 340 (2004) 95-101.

[5] E. P. Bonges, On a q-generalization of circular and hyperbolic functions, J. Phys. A, 31 (1998) 5281-5288.

[6] L. Carlitz, A degenerate Staudt-Clausen theorem, Arch. Math. (Basel), 7 (1956) 28-33.

[7] R. Dere, Y. Simsek and H. M. Srivastava, A unified presentation of three families of generalized Apostol type polynomials based upon the theory of the umbral calculus and the umbral algebra, J. Number Theory, 133 (2013) 3245-3263.

[8] K. Dilcher and C. Vignat, General convolution identities for Bernoulli and Euler polynomials, J. Math. Anal. Appl., 435 (2016) 1478-1498.

[9] Y. He, S. Araci, H. M. Srivastava and M. Acikgoz, Some new identities for the Apostol-Bernoulli polynomials and the Apostol-Genocchi polynomials, Appl. Math. Comput., 262 (2015) 31-41.

[10] D. S. Kim, T. KIm And J.-J. SeO, Umbral calculus associated with new degenerate Bernoulli polynomials, J. Comput. Anal. Appl., 22 (2017) 831-840.

[11] D. Lim, Some identities of degenerate Genocchi polynomials, Bull. Korean Math. Soc., 53 (2016) 569-579.

[12] H. Ozden, Y. Simsek And H. SRIVastava, A unified presentation of the generating functions of the generalized Bernoulli, Euler and Genocchi polynomials, Comput. Math. Appl., 60 (2010) $2779-2787$.

[13] S. Roman, The theory of the umbral calculus. I, J. Math. Anal. Appl., 87 (1982) 58-115.

[14] S. Roman, The umbral calculus, Pure and Applied Mathematics, 111. Academic Press, Inc., New York, 1984.

[15] Y. SimseK, Complete sum of products of $(h, q)$-extension of Euler polynomials and numbers, J. Difference Equ. Appl., 16 (2010) 1331-1348.

[16] H. M. Srivastava, B. Kurt And V. Kurt, Identities and relations involving the modified degenerate Hermite-based Apostol-Bernoulli and Apostol-Euler polynomials, Rev. R. Acad. Cienc. Exactas Fís. Nat. Ser. A Mat. RACSAM, 113 (2019) 1299-1313.

[17] J. Szmidt, J. URbanowicz And D. ZAGIER, Congruences among generalized Bernoulli numbers, Acta Arith., 71 (1995) 273-278.

[18] C. Tsallis, Possible generalizations of Boltzmann-Gibbs statistics, J. Stat. Phys., 52 (1988) $479-487$. 
[19] C. Tsallis, What are the numbers that experiments provide?, Quimica Nova, 17 (1994) 468471.

[20] P. T. Young, Degenerate Bernoulli polynomials, generalized factorial sums, and their applications, J. Number Theory, 128 (2008) 738-758.

[21] D. ZAGIER, A modified Bernoulli number, Nieuw Arch. Wiskd. (5), 16 (1998) 63-72. 